\title{
Care Innovations: Integration of Flash Glucose Monitoring in the Transition of Care from Inpatient to Outpatient
}

\author{
Radhika Jaiswal ${ }^{1}$, Sharon Zuniga ${ }^{1}$, Mulugeta Sarbanes ${ }^{2}$, Alyson K Myers ${ }^{1,2,3,4^{*}}$ \\ ${ }^{1}$ Department of Medicine, Division of Endocrinology, North Shore University Hospital, Manhasset, NY, USA \\ ${ }^{2}$ Donald and Barbara Zucker School of Medicine at Hofstra/Northwell, Hempstead, NY, USA \\ ${ }^{3}$ Institute for Health System Science, Northwell Health, Manhasset, NY, USA \\ ${ }^{4}$ The Feinstein Institute for Medical Research, Manhasset, NY, USA
}

Submission: June 04, 2021; Published: July 12, 2021

*Corresponding author: Dr. Alyson Myers, North Shore University Hospital, Manhasset, NY 10021, Tel: 516-708-2540, Fax: 516-708-2573, Email: amyers@northwell.edu

Abstract

In 2020, the United States had an increase in hospitalizations due to SARS-CoV-2 (COVID-19). Many of these patients also had co-morbid diabetes. In order to minimize patient - provider contact the Food and Drug Administration (FDA) granted emergency approval for inpatient use of continuous glucose monitors (CGM). Implementing the use of new technology during a pandemic does not come without challenges, as a result it was deferred to time of discharge. In this piece we address the patient, nursing and provider benefits and pitfalls of integrating CGM in patient care for persons with diabetes who have never used CGM technology in the past.

Keywords: Type 2 Diabetes; CGM; Inpatient Diabetes; COVID-19; Transitions of Care

\section{Introduction}

Hospitalization is common in persons with diabetes as roughly $25-30 \%$ of inpatients have a diagnosis of diabetes [1]. Glycemic control during the admission is of paramount importance, as inpatient hyperglycemia is associated with increased mortality, longer length of stay and increased hospital cost [2,3]. Monitoring of inpatient glucose values has traditionally been done with point of care (POC) testing, but with the onset of SARS-CoV-2 (COVID-19) many institutions were forced to use alternative means to collect glucose values [4]. Due to the FDA approval of inpatient continuous glucose monitoring (CGM) for emergency use [5], hospitals were able to use these devices to decrease staff contact with patients. There have been no large randomized-controlled trials to examine the reliability and efficacy use of CGM in the inpatient setting. The few trials which have been done have been mostly observational, 35 patients or less (except one which had 125 patients); and have demonstrated good reliability between POC testing and CGM values [6]. In addition, nocturnal hypoglycemia was detected better in CGM users compared to those who simply had POC testing [6].

The FreeStyle Libre is a flash glucose monitor, a subset of CGM, which measures glucose levels in the interstitial fluid when the user scans the sensor. Unlike some of the older CGM models, the FreeStyle Libre does not require calibration with blood glucose testing. Studies have shown that the use of this technology can either improve or make little impact on glycemic control as measured by Hemoglobin A1c and glucose variability [7-10]. Use of this technology in the outpatient setting has been associated with both improved patient satisfaction [8] and increased glucose monitoring [4].

In late April 2020, we implemented a feasibility study examining the integration of the FreeStyle Libre during the transition of care from the inpatient to outpatient settings. Patients with Type 2 diabetes who were not pregnant and were discharged on at least 3 insulin shots/day making them eligible for outpatient insurance coverage were invited to participate in this pilot. Patients who had cognitive impairment, CKD 4, 5 or ESRD or adhesive allergies were excluded. All patients who met the inclusion criteria were consented and trained by the study team. Patient training involved a step-wise approach: 1) demonstrating to the patient how to place the sensor 2) downloading the Libre Link app on the smart phone or setting up the reader 3 ) educating the patient to scan the device correctly and in timely fashion and 4) enabling data sharing feature in Libre Link app to track usage 
and glycemic control remotely.

While implementing this feasibility study, we learned many lessons at the patient, nursing, and provider levels.

\section{Lessons Learned at the Patient Level}

There were three main issues that arose with patients: poor adhesion of the sensor, software incompatibility and involvement of the patient's outpatient support team. Adhesion of the Libre was an issue for several participants and the reason that some persons declined enrollment. A few used the FreeStyle Libre in the past but did not like it as sensor kept falling off, so they were not willing to try again. A few patients enrolled also had issues with the sensor falling off as well. One was readmitted and he was retrained on how to put the sensor on and was encouraged to use extra adhesive if he still had issues in the future. Another participant initially also expressed intense apprehension regarding the sensor's adherence capabilities based on frustrating experiences in the past and cited personal "robust sleeping habits" that he believed would contribute to adhesion problems. However, after a few nights, multiple showers, and a few instances where the sensor bumped against a wall or another hard surface, he was pleasantly surprised by the sensor's durability and adherence. Throughout the entirety of the study, there were zero documented instances of incidental removal of any FreeStyle Libre.

When attempting to connect patients to the LibreLink, we encountered numerous technical issues with their phones including having non-smart phones or having smart phones incompatible with the LibreLink application (i.e., Motorola phone). The FreeStyle LibreLink app is only compatible with certain mobile devices such as: iPhone 7, Samsung Galaxy S5 or later, Google Pixel, Pixel 2, Pixel 2 XL; Nexus 5X, 6P OnePlus 5T and operating systems iOS 11 or later or Android 5 or later. Another challenge is that the LibreLink software is not compatible with the iPhone IOS14 Beta. For instance, one patient had to roll back to IOS13.6 in order to download the application. In a few instances, despite having compatible phones, participants were unable to download the LibreLink application because they either did not have the proper sign-in credentials for their Google Play or Apple App Store accounts, or their phones did not have adequate storage to download the LibreLink application. Providers also encountered technical difficulties while downloading the LibreLink app on smart phones while training patients. In one case, a few hours after the sensor were placed, the patient kept getting an error message to remove the sensor and that added more to the technical difficulties. Another patient successfully connected to her phone but on the day after discharge she received a message saying that she needs to apply a new sensor.

Additionally, some patients preferred a family member involved in their medical care to be present at the time of consent and training, requiring timely coordination between provider and family members. When family members were present during the consent and training process, there was generally an increased interest in the CGM technology. In more than one instance, family members contributed to the consenting process by reiterating the benefits of the technology and by mitigating the patients' concerns. Additionally, a few participants' family members independently discovered other potential benefits of the technology, including the LibreLink application's ability to share glucose readings with other family members from the participant's phone. These instances highlighted additional benefits of CGM technology that extend beyond the scope of this research. For patients who strongly desired the input of family members during the consent and training process, the visitation restriction during COVID-19 proved to be a significant obstacle. Five patients who declined to participate in the study preferred to talk with family or their diabetes provider first before committing to a novel method of monitoring blood sugars. These instances indicate the importance of coordination of care for the effective use of this technology. As this study was being done at the time of discharge, conversations with family or the outpatient provider were not always feasible. In one instance, the caregiver not the patient wanted to be involved as the caregiver was concerned that his mother was unable to use the technology due to her age.

\section{Lessons Learned at the Nursing Level}

Along with patients, nursing staff were also educated about the study to implement it successfully, but we encountered some challenges including patient refusal of POC testing and coordinating replacement of the Libre when the patient went for testing. Some patients who wore the FreeStyle Libre sensor prior to discharge refused POC glucose testing which is the current standard of care for hospitalized patients with diabetes. When patients need certain imaging tests or interventional procedures that required removal of sensor, nursing staff did not have any outlined systemwide or hospital protocols in place for these situations which lead to coordination between nursing staff, provider, and Certified Diabetes Care and Education Specialist (CDCES) to remove the sensor and replace it once imaging test was done.

Issues encountered by our CDCES included the unpredictability of discharge, unclear documentation regarding discharge plans (seen with almost all patients), and what our CDCES referred to as "trying to chase patients down to train them before discharge". Two patients were discharged after the CDCES left for the day and there was no clinical documentation nor provider communication regarding discharge. Our CDCES also experienced one patient asking her to remove the sensor saying "I do not like this, and I do not want to use this take it off. I rather stick my fingers" after application of sensor and complete CGM training.

\section{Lessons Learned at the Provider Level}

There were some challenges noted for providers as well, including trying to obtain consent and downloading patient data. We attempted to consent and train patients on the day of or the 
day before discharge. In some instances, this did not happen. One patient was supposed to leave on a Saturday, so he was consented and trained the Friday before. The patient ended up staying in the hospital three more weeks and expired. Change in discharge plans during hospitalization and readmissions posed another set of difficulties. For example, a patient was withdrawn as she was believed to be going home on basal/bolus insulin therapy but then was discharged on basal insulin and orals. Per insurance guidelines, patients need to be on 3 or more shots of insulin daily to have CGM coverage. A few patients were readmitted within a week of discharge, so their Libre use was minimal during rehospitalization. Data monitoring was done through LibreView app but frequently patients did not always scan sensor every 8 hours which resulted in loss of data continuity, and we were unable to interpret their data.

\section{Conclusion}

In conclusion, the integration of CGM in the inpatient setting at the time of discharge can be a very useful tool to improve glucose monitoring especially during a pandemic, such as COVID-19. This study identified a number of challenges at the patient, nursing, and provider level, which must be ameliorated to fulfill the significant potential of this technology.

\section{References}

1. Draznin B, Gilden J, Golden SH, Inzucchi SE, David Baldwin, et al. (2013) Pathways to Quality Inpatient Management of Hyperglycemia and Diabetes: A Call to Action. Diabetes Care 36(7): 1807-1814.

2. Newton CA, Young S (2006) Financial implications of glycemic control: results of an inpatient diabetes management program. Endocr Pract 12(Supplement 3): 43-48.

3. Umpierrez GE, Isaacs SD, Bazargan N, You X, Thaler LM, et al. (2002) Hyperglycemia: An Independent Marker of In-Hospital Mortality in Patients with Undiagnosed Diabetes. J Clin Endocrinol Metab 87(3): 978-982.

4. Shehav-Zaltzman G, Segal G, Konvalina N, Tirosh A (2020) Remote Glucose Monitoring of Hospitalized, Quarantined Patients with Diabetes and COVID-19. Diabetes Care 43(7): e75-e76.

5. Coronavirus (COVID-19) Update: FDA Issues Emergency Use Authorization for Potential COVID-19 Treatment. FDA. Published May 4, 2020. Accessed May 9, 2020. https://www.fda.gov/news-events/ press-announcements/coronavirus-covid-19-update-fda-issuesemergency-use-authorization-potential-covid-19-treatment

6. Davis G, Galindo R, Migdal A, Umpierrez G (2020) Diabetes Technology in the Inpatient Setting for Management of Hyperglycemia. Endocrinol Metab Clin North Am 49(1): 79-93.

7. Carlson AL, Mullen DM, Bergenstal RM (2017) Clinical Use of Continuous Glucose Monitoring in Adults with Type 2 Diabetes. Diabetes Technol Ther 19(S2): S4-S11.

8. Beck RW, Riddlesworth TD, Ruedy K, Andrew Ahmann, Stacie Haller, et al. (2017) Continuous Glucose Monitoring Versus Usual Care in Patients with Type 2 Diabetes Receiving Multiple Daily Insulin Injections. Ann Intern Med 167(6): 365-374.

9. Haak T, Hanaire H, Ajjan R, Hermanns N, Riveline J-P, et al. (2017) Flash Glucose-Sensing Technology as a Replacement for Blood Glucose Monitoring for the Management of Insulin-Treated Type 2 Diabetes: a Multicenter, Open-Label Randomized Controlled Trial. Diabetes Ther 8(1): 55-73.

10. Ontario Health (2019) Flash Glucose Monitoring System for People with Type 1 or Type 2 Diabetes: A Health Technology Assessment. Ont Health Technol Assess Ser 19(8): 1-108.

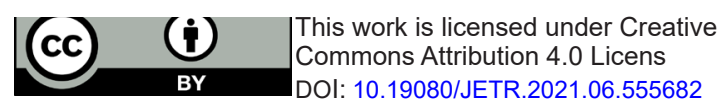

